\title{
Essais
}

ESSAIS

Revue interdisciplinaire d'Humanités

Résister entre les lignes

\section{Poésie par effraction : textes traduits de José María Gómez Valero, David Eloy Rodríguez et David Franco Monthiel}

Judite Rodrigues

\section{(2) OpenEdition \\ Journals}

Édition électronique

URL : http://journals.openedition.org/essais/4771

DOI : 10.4000/essais.4771

ISSN : 2276-0970

Éditeur

École doctorale Montaigne Humanités

Édition imprimée

Date de publication : 15 juillet 2016

Pagination : 176-195

ISBN : 978-2-9544269-8-3

ISSN : 2417-4211

Référence électronique

Judite Rodrigues, «Poésie par effraction : textes traduits de José María Gómez Valero, David Eloy

Rodríguez et David Franco Monthiel », Essais [En ligne], 9 | 2016, mis en ligne le 23 octobre 2020,

consulté le 29 octobre 2020. URL : http://journals.openedition.org/essais/4771 ; DOI : https://doi.org/ 10.4000/essais.4771 


\title{
Poésie par effraction : textes traduits de José María Gómez Valero, David Eloy Rodríguez et David Franco Monthiel
}

\author{
Judite Rodrigues
}

En 1992, l'Espagne, fastueusement ripolinée dans un emballement tout à la fois spéculatif et mémoriel de "tolérance des trois cultures ", de découverte de l'autre $^{1}$ et de passion pour la vie ${ }^{2}$, l'Espagne, donc, tente de légitimer sa place au sein des démocraties occidentales en mobilisant tous les capitaux symboliques susceptibles d'être ralliés à sa cause : Quinto Centenario, Exposition Universelle de Séville, Madrid "capitale culturelle de l'Europe ", Jeux Olympiques de Barcelone, etc. Mais, au même moment, sans faire de bruit, un collectif « d'agitation et d'expression $"^{3}$ commence à prendre forme autour de projets et de performances mêlant poésie, scène, dessin, musique... Ce collectif ou "récommune $»^{4}$, La Palabra Itinerante 5 , ouvre ainsi les hostilités artistiques avec un programme ambitieux et non négociable :

Tu misión es inventar una comunicación transformadora. Es un trabajo emocional. Tu tarea es revelar (es decir, expresar lo que hondamente ya se sabe, pero es velado entre falacias, simulacros y silencios), señalar que el emperador está desnudo, que su traje es mentira, y que esa mentira está sostenida por violencias y explotaciones. Tu tarea es denunciar la herida y meter la mano en su sangre. Negar, resistir. No quieras entretener: ese trabajo es el suyo, de eso ya se encargan ellos. Nosotros estamos aquí para intentar decir y practicar verdades. Nadie dijo que fuera fácil. ${ }^{6}$

1 Le thème choisi pour l'exposition Universelle de Séville en 1992 était : «L'ère des découvertes ».

2 On se souviendra sans doute du slogan touristique des années quatre-vingt-dix : «L'Espagne a une passion : la vie".

3 «La Palabra Itinerante es un colectivo de agitación y expresión, una plataforma de acción artística y literaria, una comunidad ", http://www.soloamedias.net/quienes/palabra.html [consultation mars 2015].

4 Nous avions fait le choix de ce néologisme dans une brève présentation de ces différents collectifs : Rodrigues Judite, "Les contre-feux de la poésie espagnole contemporaine : les "récommunes" poétiques de l'engagement ", communication au congrès de la SHF, Bordeaux, juin 2015.

5 Pour une chronique de la genèse du collectif, lire : Franco Monthiel David, "La Palabra Itinerante, la palabra resistente ", 22/04/2003, http://www.rebelion.org/hemeroteca/cultura/ david210403.htm [consultation septembre 2015].

6 La Palabra Itinerante, «Usted también puede ser un poeta en resistencia. Algunos consejos útiles 
Cette citation est extraite d'un des textes programmatiques du collectif intitulé "Usted también puede ser un poeta en resistencia. Algunos consejos útiles si este es su objetivo". Il existe un deuxième texte qui pose les bases de la plateforme: "Una manera de mirar pájaros en vuelo: una aproximación a la poesía en resistencia ${ }^{7}$. Ces deux actes fondateurs sont des invites radicales à la résistance, à l'effraction et à l'émancipation.

Parmi les principaux promoteurs et acteurs de ce laboratoire d'idées, on trouve les poètes José María Gómez Valero (Séville, 1976) et David Eloy Rodríguez (Cáceres, 1976). Tous deux ont commencé à publier de façon régulière à partir de la deuxième moitié des années quatre-vingt-dix ${ }^{8}$, mais ils ont aussi à leur actif des publications collectives (en littérature jeunesse ${ }^{9}$ notamment ou sur des projets inter-artistiques ${ }^{10}$ ). Autour d'eux, le tournoiement de voix s'est fait intense : «Son poetas de la Resistencia. Y lo mejor es que no están solos. Junto a ellos se agrupa una horda multicolor de maduros jóvenes empecinados que pululan alegres y tenaces por las mismas trincheras. ;Qué orgullo, militar en esas nobles filas! ${ }^{11}$.

En 2005, c'est ensemble, également, qu'ils ont lancé la maison d'édition "Libros de la Herida " ${ }^{12}$. Dix ans d'existence et une dizaine de livres édités au compteur. Le colophon du premier recueil publié explique que la blessure qui

si este es su objetivo ", http://soloamedias.net/quienes/usted_tambien_puede.pdf [consultation mars 2014].

7 La Palabra Itinerante, "Una manera de mirar pájaros en vuelo: una aproximación a la poesía en resistencia ", http://soloamedias.net/quienes/una_manera_de_mirar.pdf [consultation mars 2014].

8 Pour David Eloy Rodríguez : Chrauf, Sevilla, Universidad de Sevilla, 1996 ; Miedo de ser escarcha, Sevilla, Qüasyeditorial, 2000 ; Asombros (en collaboration avec l'artiste peintre Miki Leal), Sevilla, César Sastre, Imagofórum, 2006 ; Los huidos, Logroño, Ediciones del 4 de Agosto, 2008 ; Para nombrar una ciudad, Sevilla, Renacimiento, 2010 ; Miedo de ser escarcha (édition actualisée), Mérida, Editora Regional de Extremadura, 2012; Lo que iba diciendo, Cáceres, Ediciones Liliputienses, 2012 (sélection de poèmes) ; Desórdenes, Madrid, Amargord, 2014 ; Poesía desde el espacio, Mérida, De La Luna Libros, 2014.

Pour José María Gómez Valero : Miénteme, Sevilla, Qüasyeditorial, 1997 ; El Libro de los simulacros, Ayuntamiento de Lepe, 1999 ; Travesía encendida, Madrid, Ediciones Vitruvio, 2005; Lenguajes (en collaboration avec l'artiste peintre José Miguel Pereñíguez), Sevilla, César Sastre, Imagofórum, 2007 ; Los Augurios, Barcelona, Icaria Editorial, 2011.

9 García Argüez Miguel Ángel, Gómez Valero José María, Rodríguez David Eloy, Celaya Amelia, Cosas que sucedieron (o no), Oviedo, Cambalache, 2013 ; García Argüez Miguel Ángel, Gómez Valero José María, Rodríguez David Eloy, Celaya Amelia, Este loco mundo, 17 cuentos, Oviedo, Cambalache, 2010.

10 Colectivo La Palabra Itinerante, Su mal espanta, Sevilla, Libros de la Herida, 2014. Un CD-livre qui réunit les textes de David Eloy Rodríguez et José María Gómez Valero, avec les dessins de Patricio Hidalgo et la musique et le chant de Danie Mata et Enrique Mengual.

11 Porlan Alberto, "Antorchas en la noche ", prologue à Gómez Valero José María, Pereñíguez José Miguel, Lenguajes, op. cit., 2007, p. 8.

12 C'est dans la collection "Poesía en Resistencia " que l'on trouvera sans doute les matières les plus inflammables pour l'insurrection des consciences. 
donne son nom à cette fabrique éditoriale dit à la fois le désarroi, la fragilité mais aussi le combat : "Libros de poemas para pensar el mundo, palabras que cuentan la herida abierta del vivir, su horror y su milagro ${ }^{13}$. Performances artistiques qui réinvestissent les espaces publics, rencontres littéraires, ateliers pédagogiques d'écriture, création d'une maison d'édition indépendante, etc., toutes ces entreprises, tous ces événements, sont autant de "réponses politiques ${ }^{14}$ qui mêlent l'activité créatrice et l'action ${ }^{15}$.

Dans le panorama actuel de la poésie espagnole, ces voix dissidentes sont parfois cataloguées sous les étiquettes de poésie " de la conscience critique ", poésie "néo-sociale ", ou bien encore "poésie du conflit ". Dans son étude, Poesía de la conciencia crítica (1987-2011) ${ }^{16}$, Alberto García-Teresa, à la fois analyste et insider du mouvement, recense une trentaine de poètes. On voudrait ici simplement, avant de donner à lire quelques-uns de leurs textes traduits, présenter brièvement l'œuvre de trois d'entre eux qui participent à l'aventure collective de La Palabra Itinerante : José María Gómez Valero, David Eloy Rodríguez et David Franco Monthiel (Cádiz, 1976) ${ }^{17}$. Trois poètes, trois voix " réfractaires " selon l'expression de René Char, qui ont en commun une écriture avide et batailleuse semblable à la respiration du noyéc ${ }^{18}$. Leur programme poétique pourrait se résumer à ces deux mots empruntés à l'œuvre de l'un d'entre eux : " canto y demolición " ${ }^{19}$. Les mots, embrasés

13 Del Pozo Pedro, Todas las puertas abiertas, Sevilla, Libros de la herida, 2005, s. p.

14 "Il va de soi que la prolifération des structures éditoriales autonomes, la multiplication des modes d'intervention publique, la culture de réseaux (en place du fonctionnement groupal), l'appropriation de tous les outils de la communication contemporaine, leur usage direct ou décalé, détourné, etc., tout cela participe d'une réponse politique à la pression du contexte ", Gleize JeanMarie, "Opacité critique ", in Bailly Jean-Christophe, Gleize Jean-Marie, Hanna Christophe... [et al.] Toi aussi, tu as des armes : poésie \& politique, Paris, La Fabrique, 2011, p. 31.

15 L'achevé d'imprimé du deuxième recueil édité revendique ainsi très clairement cet ancrage dans le politique : «El nueve de octubre de 2007 se terminó de imprimir Compañero enemigo del poeta Juan Antonio Bermúdez. John Lennon cumplía en los corazones de muchos sesenta y siete años e Irak continuaba desangrándose por aquellos a quienes la vida no importa nada, los asesinos en nombre del Dios dinero, del Dios petróleo, del Dios poder, del Dios-dogma. He aquí el tiempo de los asesinos escribió Rimbaud. Pese a todo, multitudes en todo el mundo seguían diciendo, escribiendo, haciendo su no consentimiento, su respiración para la vida, su alegría, su amor ", Bermúdez Juan Antonio, Compañero enemigo, Sevilla, Libros de la Herida, 2007, s. p.

16 García-Teresa Alberto, Poesía de la conciencia crítica (1987-2011), Madrid, Tierradenadie ediciones, 2013.

17 Bibliographie de David Franco Monthiel : Renta básica de olvido, Huelva, Ayuntamiento de Lepe, 2004 ; Aforos completos y otros mínimos aforismos, Logrońo, Ediciones del 4 de agosto, 2007 ; Las Cenizas de Salvochea, Tenerife, Baile del Sol, 2008 ; Apuntes de la servidumbre, Cuadernos caudales de poesía, Fundación Inquietudes, 2009 ; Yuri Gagarin que estás en el cielo, Diputación de Cádiz, 2011 (nouvelles) ; Libro de la servidumbre, Valencia, Germanía, 2011.

18 On se souviendra qu'en 1929, dans une lettre adressée à Paul Éluard, René Char définissait l'écriture comme "de la respiration de noyé », Char René, Euvres complètes, Paris, Gallimard, 1983.

19 "Canto y demolición " est le titre d'une section du recueil Para Nombrar una ciudad de 
par la rougeur de l'espérance, y interrogent irrémédiablement la capacité de l'homme à se décider pour le bonheur. La force d'invention des poèmes, leur tranchant aphoristique donnent une puissance particulière à ces carnets poétiques de «buissonnage " qui invitent à l'émancipation quotidienne et à la sortie de la résignation. L'ironie, l'humour, l' " appel du mur » sont quelquesunes des marques de cette nouvelle poésie engagée que nous voudrions mettre au jour dans cette brève présentation.

\section{Une faim de « nous » : la fabrique du commun}

L'activisme dans le collectif La Palabra Itinerante a sans doute nourri une habitude et une pratique du commun en poésie. Un commun incarné dans l'écriture par une première personne du pluriel qui revendique hautement sa place. Le "nous " en poésie n'est pas une collection de « je ", car on le sait, il y a plus dans le tout que dans la somme des parties ${ }^{20}$. Le "nous " y est donc une puissance, un rempart, une ligne de front : "Somos de aquí / Somos del aquí $»^{21}$, "La muerte está frente a nosotros $»^{22}$, "Vamos juntos " ${ }^{23}$. Ce "nous " s'inscrit dans les récits et chroniques de nos temps modernes : peurs, violences, résignations, combats menés... Le recueil Libro de la Servidumbre de David Franco Monthiel contient par exemple un appareil de notes commentant les éléments contextuels évoqués : Wafa Idris, première femme kamikaze palestinienne de l'histoire, Satar Jabar, prisonnier d'Abu Ghraib dont la photo a fait le tour du monde, Halliburton, entreprise multinationale parapétrolière dont les enjeux économiques sont liés aux différentes guerres menées à la fin $\mathrm{du} \mathrm{XX}^{\mathrm{e}}$ siècle, etc. En travaillant ces matériaux, les poèmes pourraient alors s'apparenter à des "lieux de mémoire ${ }^{24}$ qui disent furieusement combien

David Eloy Rodríguez. C’est aussi le titre choisi pour l'anthologie italienne Canto y demolición: 8 poetas españoles, Pesaro, Thauma, 2013, qui réunit huit poètes espagnols contemporains dits de la " conscience critique".

20 Dans le préambule à la plaquette de poésie " Un zumo de tres sabores o un itinerario compartido ", la présentation du collectif insiste sur cette idée de la puissance du commun : " La Palabra Itinerante es un colectivo de agitación y expresión, una plataforma de acción artística y literaria, una comunidad. Lo forman escritores/as y artistas, con percepciones estéticas y éticas en común, que suman esfuerzos y comparten camino y aprendizajes en el deseo de participar en la construcción social, no sólo a través de la creación de cada texto (bien de forma individual, bien de forma conjunta), sino también mediante (otras) prácticas sociales concretas que acerquen el arte a las gentes y lo ofrezcan como herramienta que puede ayudarnos a vivir más vivamente: a comprender mejor, a sentir, a transformar, y que propicien espacios y tiempos oportunos para la reflexión crítica, para el encuentro y el diálogo ", Colectivo La Palabra Itinerante, "Un zumo de tres sabores o un itinerario compartido ", Cuadernos caudales de poesía, II, Fundación Inquietudes, 2009, s. p.

21 Rodríguez David Eloy, Desórdenes, op. cit., p. 72.

22 Ibid, p. 73.

23 Ibid, p. 75.

24 "Les lieux de mémoire ce ne sont pas ce dont on se souvient mais là où la mémoire travaille ", 
l'indifférence est haïssable ${ }^{25}$. Les poèmes invitent à la colère, à l'indignation en fâchant "tout rouge " ${ }^{26}$ les lecteurs : "Aprender del lenguaje / a construir / y a decir no ${ }^{27}$, «Vivir furioso / pero nunca cegado por el odio ${ }^{28}$, «Teoría de la desobediencia en cuatro palabras: No. No. Y no ${ }^{29}$.

L'empuissantisation de la multitude se construit dans une identité tout à la fois singulière et plurielle : "El privilegio de compartir temblor y alegría, / uno más en la multitud, / cuerpo entre los cuerpos, / presencia. / La avidez de vivir nos convoca $"^{30}$. Cette citation est tirée du dernier recueil de David Eloy Rodríguez, La poesía vista desde el espacio. Dans cette œuvre, il est fondamentalement question de ce qu'est la poésie et de ce qu'elle n'est pas ${ }^{31}$. Son auteur discrimine sans longs ambages les poètes des prestidigitateurs de la poésie ${ }^{32}$ en démontant avec ironie les préceptes de « La Organización Mundial de la Poesía " ${ }^{33}$. Mais il est parfois tentant pour l'exégète d'extrapoler et de voir dans les spécificités du métier de poète, le métier du vivre. Ces deux vers par exemple, "Sorprende la tenacidad con la que nos entregamos / a desperdiciar las ocasiones ${ }^{34}$, semblent nous inviter, tous, dans cette première personne grammaticale du pluriel, à prendre conscience du temps maladroitement perdu dans les entreprises inutiles. Plus loin, l'invitation à l'émancipation par la mise en branle de la force de la multitude se complète : "No estamos derrotados: / estamos vivos ${ }^{35}$. Une dynamique est ainsi lancée par la création de ce corps collectif puissamment constitué dans ce "nous " revendiqué.

Le recueil de David Eloy Rodríguez qui pose le plus puissamment cette force d'un " nous " collectif combattif est sans doute Miedo de ser escarcha. La dédicace à l'édition de 2012 est à elle seule révélatrice : "Este libro es por y para vosotras y vosotros: de vuestra/nuestra sangre quiere estar hecho ${ }^{36}$. Tout le recueil est porté par un élan du commun. Dans les parties aux titres évocateurs, "Nosotros, todos nosotros » ou " La hermandad de la sangre ", on lira cette affirmation de la puissance de l' « être-ensemble » et la fédération d'un

Nora Pierre, Les lieux de mémoire, t. I, La République, Paris, Gallimard, 1984, p. 17.

26 Sans doute n'y a-t-il pas là de hasard chromatique...

27 Rodríguez David Eloy, La poesía vista desde el espacio, op. cit., p. 39.

28 Franco Monthiel David, Las Cenizas de Salvochea, op. cit., p. 25.

29 Ibid, p. 29.

30 Rodríguez David Eloy, La poesía vista desde el espacio, op. cit., p. 74.

31 Le poème " Tiempo vivo " est particulièrement significatif, Rodríguez David Eloy, La poesía vista desde el espacio, op. cit., p. 40.

32 "No hay que confundir la poesía con la poesía ", Rodríguez David Eloy, La poesía vista desde el espacio, op. cit., p. 80.

33 Ibid., p. 66.

34 Ibid., p. 56.

35 Ibid., p. 61.

36 Rodríguez David Eloy, Miedo de ser escarcha (édition actualisée), op. cit., p. 11. 
corps politique autour d'une colère. À l'ère de ce que l'on appelle "l'individualisme " ou le "communautarisme ", une nouvelle option est ici proposée : celle d'un commun non substantiel fédéré autour de l'idée de la résistance et de l'émancipation.

\section{Un retournement du monde : entre détournement et satire}

Le détournement, qui est sans doute un des traits dominants de l'œuvre de David Franco Monthiel et principalement dans Las Cenizas de Salvochea, consiste en la réappropriation d'un matériel en renversant et brisant des chaînes de signifiance solidement ancrées. Le détournement est ici le plus souvent parodique, subversif et résolument militant. Cette mise à distance critique des objets détournés est héritée des techniques transformationnelles théorisées et pratiquées par Guy Debord ${ }^{37}$. Dans leur article fondateur, «Mode d'emploi du détournement ${ }^{38}$, les tout jeunes lettristes Wolman et Debord expliquaient ainsi les implications politiques de telles expériences : développement d'une guerre corrosive et iconoclaste au service d'un projet politique d'émancipation par la mise à mal des notions d'autorité, de propriété privée et la création d'une communauté, d'un « communisme » littéraire ${ }^{39}$.

Le cogito ergo sum est ainsi par exemple détourné en cet énoncé "Piensa. Luego, insiste $»^{40}$ qui montre les possibilités, dans l'exercice d'une pensée critique et active, de l'impertinence, de la persévérance et de la contestation. Ces vers qui disent le choix de la rétivité plutôt que la docilité, l'insoumission plutôt que la résignation, se présentent comme la formulation "détotemisée " du préambule à l'entrée en rébellion. L'altération consiste ici en un transfert vers une forme d'impératif à la deuxième personne grammaticale ("Piensa ») ${ }^{41}$ et une modification du deuxième verbe jouant sur un effet de paronomase in abstentia (" existir ", " insisitir »). L'énoncé " Piensa. Luego insiste " relève donc du slogan affiché et comme placardé. On retrouvera ce

37 David Franco Monthiel revendique cette filiation et la porte en note d'un des poèmes de ce recueil : " "Détournement Cohen" hace referencia al concepto situationista de "tergiversación" (distorsionar el significado y uso original de palabras, frases y objetos para producir un efecto crítico)", Monthiel David Franco, Las Cenizas de Salvochea, op. cit., p. 27.

38 Debord Guy-Ernest, Wolman Gil J., " Mode d'emploi du détournement ", in Debord Guy, Euvres, Paris, Gallimard, 2006. Cet article est d'abord paru dans le $\mathrm{n}^{\circ} 8$ de la revue Lèvres Nues (mai 1956) sous la signature d'Aragon et André Breton.

39 "Dans son ensemble, l'héritage littéraire et artistique de l'humanité doit être utilisé à des fins de propagande partisane. Il s’agit, bien entendu, de passer au-delà de toute idée de scandale. [...] A vrai dire, il faut en finir avec toute notion de propriété personnelle en cette matière ", Debord Guy, Euvres, op. cit., p. 221.

40 Franco Monthiel David, Las Cenizas de Salvochea, op. cit., p. 28.

41 Les hypothèses qui sont développées cet article (celles de l'écriture du slogan et de l'« appel du mur») nous invitent raisonnablement à comprendre ces formes verbales comme des impératifs et non des troisièmes personnes du singulier au présent de l'indicatif. 
même cogito pareillement détourné chez d'autres poètes dits « de la conscience critique ", avec un même effet déconstructeur qui réveille les pensées réfractaires : "Pienso, luego molesto si lo digo » ${ }^{42}$ écrit Jorge Arévalo. "Pienso luego sur $"{ }^{43}$ complète Isabel Pérez Montalbán.

Dans les détournements, ce sont aussi des textes-emblèmes qui sont parfois retravaillés. Leur manipulation, en entraînant la dissolution du sens premier, insuffle alors un nouveau potentiel poétique. C'est le cas par exemple du poème «Los trabajos temporales y los días ${ }^{44}$ qui met en place un réseau de signifiance triple en réutilisant le substrat des textes de Bécquer et d'Hésiode :

LOS TRABAJOS TEMPORALES Y LOS DÍAS

Volverán las dulzonas hojaldrinas

Aseveró

el poeta

hambriento

Les tourments du poète romantique devenu poète intérimaire et affamé ne manquent pas de malice. Mais ce sourire du premier abord dans le passage au prosaïque des " oscuras golondrinas " devenues "dulzonas hojaldrinas " s'efface vite dans l'échelonnage d'un vers qui pose l'inaccessible, la dépossession, le manque. C'est le monde du travail qui est ici caractérisé dans ses pénuries et ses défaillances. Et l'humour est particulièrement grinçant dans le télescopage entre l'univers intimiste du romantisme poétique et l'univers contemporain de structures du travail destructrices. L'ironie et le pastiche sont sans doute des armes à puissance de feu plus élevée ${ }^{45}$. La capacité offensive de ces missiles poétiques tient à la justesse du rapprochement, au caractère cinglant du mot d'esprit, à l'impertinence de l'agudeza : "Cordero de dios : / Quita el mercado del mundo » ${ }^{46}$, "Lo real no es l'Oréal ${ }^{47}$.

De l'humour, de l'ironie, du grinçant... mais aussi de la satire, hautement explosive, comme dans le poème "Nación ", " Múltiple, pequeña y libérrima ${ }^{48}$, qui fait voler en éclat la devise "Una, grande y libre », propagée ad

42 Jorge Arévalo in Voces del Extremo II, Poesía y conciencia, Moguer, Fundación Juan Ramón Jiménez, 2000, p. 72.

43 "El sur también existe, que dice Benedetti. / No sé si existo, pero si existo soy el sur. / Pienso luego sur ", Isabel Pérez Montalbán in Voces del Extremo II, Poesía y conciencia, op. cit., p. 143.

44 Franco Monthiel David, Las Cenizas de Salvochea, op. cit., p. 65.

45 "Il va de soi que la tendance au pastiche, à la parodie, au traitement ironisé des "messages" ambiants, des codes et formats de la communication de masse, persiste et trouve en permanence à se renouveler. C'est d'ailleurs là une des multiples façons dont le politique trouve aussi à se réintroduire, de façon plus ou moins directe, dans la production poétique, ou postpoétique, aujourd'hui ", Gleize Jean-Marie, "Opacité critique », in Bailly Jean-Christophe, Gleize JeanMarie, Hanna Christophe... [et al.] Toi aussi, tu as des armes : poésie \& politique, op. cit., p. 34.

46 Franco Monthiel David, Las Cenizas de Salvochea, op. cit., p. 21.

47 Ibid., p. 29.

48 Ibid., p. 28. 
nauseam pendant l'Espagne franquiste. Le poète démolit et desserre les liens de cette définition de la nation étriquée, xénophobe et arrogante. C’est aussi parfois un coup de canif qu'il porte au langage, et il ne s'agit pas là seulement d'une description métaphorique. Les mots sont sectionnés, découpés, pour faire surgir un sens nouveau : "Venceremos [...] Ven. Seremos " ${ }^{49}$. De la même façon, il sectionne les vers du poème "La sajadura del afilador " ${ }^{50}$ comme pour exhorter à simplement couper les fils de pantins qui nous soutiennent, rappelant ainsi le Discours de la servitude volontaire de La Boétie et son principe d'action : "Soyez résolus à ne plus servir, et vous voilà libres " ${ }^{51}$.

Ces détournements fonctionnent sans doute comme autant de rectifications, de correctifs apportés à des énoncés parfois éculés, épuisés, sclérosés. Entre distanciation, réappropriation et refondation, la technique du détournement ébranle les fétiches du langage et appelle à la puissance de pensée. Il est aussi à noter que ce procédé d'écriture, qui implique la mise en résonnance de différentes strates de signifiance, invite le lecteur à un exercice actif de décodage et requiert de lui une attitude critique.

\section{L'écriture qui appelle le mur : mot d'ordre et slogan}

Dans la tradition de la pensée critique, la théorie appelle la pratique et le savoir appelle l'action. Ici, de la même façon, l'écriture prépare le geste et le livre cristallise sa puissance dans les consciences et dans la rue. On retrouvera, dès lors, à de nombreuses reprises une poésie qui se fait mot d'ordre. La consigne, qui énonce une règle d'action, se donne par exemple sur le mode de l'impératif négatif : "Nunca sacrifiques los odios / en las elecciones del miedo [...] Nunca olvides la música de las trincheras [...] Nunca dejes de oír / la sinfonía insurrecta en la profundidad $"$, , « No te pongas de rodillas / salvo por piedad. / No calles / sino por piedad " $^{53}$, «No te confíes al pulso / de un cirujano epiléptico ; / No rellenes el formulario de la libertad $~{ }^{54}$. David Eloy Rodríguez fait aussi le choix de la puissance des infinitifs associés : «Suspender, interrumpir. / Desencerrar. / Hacer presente. / Señalar. / Interrogar. / Negar. / Acompañar. / Construir " ${ }^{55}$. José María Gómez Valero enrichit la liste : "Amar sin daño, / amar sin dueño. / Saber reír, / saber curar $»^{56}$. Il ne s'agit sans doute pas là d'un

49 Ibid., p. 30. La traduction de ce vers apparaît comme particulièrement malaisée.

50 Ibid., p. 36.

51 «Soyez donc résolus à ne plus servir et vous serez libres. Je ne veux pas que vous le heurtiez ni que vous l'ébranliez, mais seulement ne le soutenez plus, et vous le verrez, comme un grand colosse dont on dérobe la base, tomber de son propre poids et se briser ", De la Boétie Étienne, Le discours de la servitude volontaire, Paris, Payot, 1976, p. 202-203.

52 Franco Monthiel David, Libro de la servidumbre, op. cit., p. 61.

53 Rodríguez David Eloy, Miedo de ser escarcha (édition actualisée), op. cit., p. 54.

54 Ibid, p. 55.

55 Rodríguez David Eloy, La poesía vista desde el espacio, op. cit., p. 68.

56 Gómez Valero José María, Los Augurios, op. cit., p. 31. 
catéchisme, moins encore d'une morale auréolée de devoirs sacrés, mais bien de propositions d'action pour la révolution des esprits. L'invite à l'action dit ici sans conteste le rejet de la logique du renoncement et de l'abandon. Ces impératifs grammaticaux appellent à soumettre la réalité et les théories dominantes au tamis du jugement.

On retrouvera ainsi mots d'ordre, revendications, dénonciations, protestations dans des vers qui semblent répondre aux normes sémantiques et rhétoriques du slogan et du graffiti. Des vers, en somme, qui appellent les murs comme dans le titre "Muro con inscripciones desvaídas " ${ }^{57}$ qui fait référence au recueil de Jorge Riechmann, Muro con inscripciones ${ }^{58}$. La Brigada Poética en Acción répond d'ailleurs à cet appel par des micro-performances qui consistent à afficher dans les espaces publics certains de ces courts aphorismes tantôt provocateurs tantôt désarmants ${ }^{59}$. Les vers viennent ainsi à la rencontre de ceux qui prennent le temps de la halte ${ }^{60}$.

L'esprit de contestation se lit sur les murs bariolés de graffitis et de slogans. On déchiffrera dans ces palimpsestes poétiques, certains des slogans scandés et écrits en Mai 68. Autant de formules qui font partie du patrimoine des illusions révolutionnaires lyriques. Ainsi, le "Soyez réalistes, demandez l'impossible ", qui disait le refus de l'allégeance au principe de " la force des choses ", $\mathrm{du}$ " there is no alternative ", subit un retournement et devient "Sé utópica. [...] Y pide lo posible ${ }^{61}$ :

BAJO LOS ADOQUINES, LOS ADOQUINES

Sé utópica, amiga.

Y pide lo posible.

Aunque parezca

que está permitido.

57 Franco Monthiel David, Las Cenizas de Salvochea, op. cit., p. 28-30.

58 Riechmann Jorge, Muro con inscripciones, Barcelona, DVD Ediciones, 2000.

59 http://www.asociacionumbrales.blogspot.fr/ [consultation septembre 2015]. Jean-Marie Gleize parle ainsi d'une véritable occupation de l'espace public :

Ils s'approprient la langue de l'ennemi pour mieux s'insinuer dans ses réseaux de communication, pervertir ou détourner ses messages, ses systèmes de figuration, etc. D’où la métaphore du virus - supposé invisible et indétectable, d'autant plus dangereux et efficient qu'il n'est pas repérable. [...] le choix du modèle " méta-usage ", qui se sert des formes mêmes des langages dominants pour en faire la matière première d'une écriture poétique critique qui, au contraire des positions du " contre-usage " va pouvoir revendiquer, comme lieu d'intervention et d'action, l'espace public - panneaux publicitaires, écrans vidéos, posters, etc.

Gleize Jean-Marie, "Opacité critique ", in Bailly Jean-Christophe, Gleize Jean-Marie, Hanna Christophe... [et al.] Toi aussi, tu as des armes : poésie \& politique, op. cit., p. 39-40.

60 De la poésie à coups d'aérosol, c'est aussi ce fait que l'artiste grapheur Neorrabioso quand il inscrit ses vers sur les murs de la capitale espagnole. Il conviendrait aussi de mentionner l'objet poétique Skinny Cap, qui mêle poésie, graphitti et pencils, publié par Libros de la Herida: Asunción Silva Martha, Skinny Cap, Sevilla, Libros de la Herida, 2014.

61 Franco Monthiel David, Libro de la servidumbre, op. cit., p. 59. 
L'enseignement que délivre ce poème est en soi une micro-révolution des esprits : demandons ce que nous avons déjà, vivons et désirons ce que nous possédons ${ }^{62} \ldots$ On reconnaîtra peut-être ici, la pénétrante sagesse de cette jeune-fille âgé d'à peine seize ans, le personnage de Juliette dans le drame de Shakespeare, qui expliquait : «Et pourtant je ne souhaite que ce que j'ai ${ }^{63}$.

Les compositions resserrées, les formes brèves, condensées, totalisantes, celles qui se rapprochent de l'aphorisme, celles qui s'adaptent aux exigences du slogan, sont nombreuses ${ }^{64}$. C'est sans doute une des caractéristiques de ces écritures de la " conscience critique » dont l'objectif est d'éveiller les consciences.

\section{Poésie balistique : «breverías », le parti pris minimal}

Le mode d'action du poète en résistance avait été théorisé par La Palabra Itinerante de la façon suivante : «El método más adecuado es probablemente el de la guerrilla: incursiones rápidas en territorio hostil para cubrir los objetivos, y luego regresar a terreno seguro ${ }^{65}$. David Eloy Rodríguez évoque à nouveau ce procédé quand il donne pour titre à une de ses compositions : "Golpe certero y rápida huida ${ }^{66}$. La brièveté, la précision et la puissance de frappe sont sans doute une des forces de cette poésie qui s'engage. On se laisserait sans doute volontiers tenter par l'imprudence du néologisme pour nommer "brevería " cette poésie aphoristique. Il y a là, en effet, quelque chose de l'ironie et de l'humour de la greguería ${ }^{67}$. L'esprit sourit par exemple aux jeux de mots dans ces vers de José María Gómez Valero qui construisent un réseau d'analogies suggestives : "Si la herida es herencia de la vida, /

62 On pourrait donner un autre exemple de ce corpus "soixante-huitard " détourné : la célèbre injonction paradoxale "Il est interdit d'interdire ». Jorge Riechmann complète ainsi avec ironie la table des commandements en formulant son "prohibido imponer" (Jorge Riechmann, Futuralgia: poesía reunida, 1979-2000, Madrid, Calambur, 2011, p. 525). Avec ce slogan, le champ d'action est plus ample, la contestation plus globale. En effet, si l'interdiction élimine une option ("ne fais pas cela »... mais il reste encore mille choses qu'il est possible de faire), l'obligation, elle, impose une option unique (" fais cela ", aucune alternative donc...). Au-delà de la remise en cause des normes castratrices ou des orthodoxies bienpensantes, ce premier commandement, "prohibido imponer ", revendique non pas une plus grande marge de liberté mais bien plutôt un espace entier de liberté.

63 Shakespeare William, Tragédies (I), Roméo et Juliette, Paris, Gallimard, 2002, p. 277.

64 Pour une première approche des formes brèves chez deux des poètes de la « conscience critique " : Rodrigues Judite, « Le maquis poétique de David Eloy Rodríguez et José María Gómez Valero (quelques notes sur la poésie en résistance) ", Les Langues Néolatines, juin 2015.

65 http://soloamedias.net/quienes/usted_tambien_puede.pdf [consultation mars 2014].

66 Rodríguez David Eloy, La poesía vista desde el espacio, op. cit., p. 88.

67 Une «brevería » engagée formulerait par exemple en quelques vers les récidives du néocolonialisme subi par l'Amérique en travaillant conjointement jugement et jeu de mot : «El aparato digestivo de América Latina / padece desde hace quinientos años / un Cáncer de Colón ", Franco Monthiel David, Aforos completos y otros mínimos aforismos, Logrońo, Ediciones del 4 de agosto, 2007. 
manar siempre hermosura es hermanar ${ }^{68}$. Les " mínimos aforismos ${ }^{69} \mathrm{de}$ David Franco Monthiel ressemblent aussi par exemple à s'y méprendre aux commentaires et flèches d'humour des dessins de presse de El Roto. Une autre image moins belliqueuse préfererait rapprocher ces compositions de l'art de l'origami. Une simple feuille, quelques plis... c'est la proposition de David Eloy Rodríguez : " estas miniaturas / estas pequeñas esculturas de pájaros [...] Pequeñas creaciones articuladas $»^{70}$.

Les poèmes de José María Gómez Valero sont sans doute ceux qui s'expriment le plus en quintessence et qui se donnent comme le résultat de distillations successives. Passés par l'alambic du langage, ils concentrent dans une densité ultime les formes les plus épurées de la poésie. Ce parti pris minimal permet l'expression d'une ivresse sans la recherche d'effet de sublime. Alberto Porlan reconnaît ici le paradoxe de la profondeur des abysses dans le dépouillement et la sobriété : «Es la victoria en toda la línea de lo sintético abisal ante lo prolijo superficial ${ }^{71}$. Quelques touches suffisent à décrire un paysage de désir comme par exemple dans le court poème au long titre «La primera hoja caída del árbol de la aurora ": " Mis dedosombras / acarician / tu espaldaluz ${ }^{72}$. Cette si brève composition capte l'épiphanie d'un moment de désir. La collision saisissante des mots y donne à deviner les effets de diffractions de lumières dans les corps effleurés. Dans l'œuvre de José María Gómez Valero, on est souvent proche du funambulisme poétique tant l'équilibre semble impeccable et le choix des mots décisif ${ }^{3}$. Cette poésie, qui veut l'intensification, travaille sans nul doute aux forces d'expansion du vivre et du désirer.

On voudrait aussi dire un mot sur les nombreuses interrogations qui ponctuent l'œuvre de José María Gómez Valero. Le poème devient le lieu où se posent les questions mais aucune réponse préalablement travaillée, équarrie, prémâchée ou prête à l'emploi n'est apportée. Avec une fausse candeur, une innocence toute enfantine, les questions sont très directement formulées : " ¿No somos todos / extranjeros en la ciudad / del ruido y del dinero ? " ${ }^{74}$. Confirmation, dénégation, opinion, jugement, etc., le lecteur est encore une fois acteur et parti pris dans cette proposition poétique ${ }^{75}$.

68 Gómez Valero José María, Pereñíguez José Miguel, Lenguajes, op. cit., p. 21.

69 Franco Monthiel David, Aforos completos y otros minimos aforismos, op. cit.

70 Rodríguez David Eloy, La poesía vista desde el espacio, op. cit., p. 101.

71 Porlan Alberto, "Antorchas en la noche ", prologue à Gómez Valero José María, Pereñíguez José Miguel, Lenguajes, op. cit., p. 6.

72 Gómez Valero José María, Los Augurios, op. cit., p. 66.

73 On renoue là avec la définition du poète donnée par Jean-Michel Maulpoix : « Funambule, le poète avance sur une corde en mesurant ses pas [...]. Penché sur Le Pont Mirabeau, tout près de s'en jeter, le danseur de corde regarde le ciel d'en bas, tel qu'au fil du fleuve il se reflète et s'ouvre "comme un abîme" au-dessous de lui ", Maulpoix Jean-Michel, Adieux au poème, Paris, José Corti, 2004.

74 Gómez Valero José María, Los Augurios, op. cit., p. 56.

75 Dans la note-préambule à l'édition actualisée de Miedo de ser escarcha, David Eloy Rodríguez 
Si, comme le pense Bernard Noël, écrire de la poésie est déjà en soi un acte de résistance ${ }^{76}$, écrire comme le font David Eloy Rodríguez, José María Gómez Valero et David Franco Monthiel, c’est alors irrémédiablement revendiquer un droit d'effraction dans la vie. La poésie dressée en " poste d'observation privilégié » ${ }^{77}$ devient un lieu de subversion où se déploie la pensée agonistique et rétive. Car il s'agit bien de déconstruire et de combattre avec les armes du langage poétique. Le détournement, cinglant et caustique, on l'a évoqué, fait acte d'analyse et disqualifie tout à la fois les prescriptions et autres recommandations produites dans les discours anesthésiants de « la langue consommée ${ }^{78}$. De même, l'écriture d'un commun à venir et à construire pose la multitude comme une force mobilisatrice. Les «breverías » et autres poèmes cursifs travaillent aussi à la survenue d'un nouvel âge de l'émancipation. La très brève anthologie qui suit cette rapide présentation sera l'occasion de donner à lire, dans sa traduction française ${ }^{79}$, quelques textes de ces trois poètes.

\author{
Judite Rodrigues \\ EA 4182 Centre Inter Langues \\ Université de Bourgogne \\ Judite.Rodrigues-Balbuena@u-bourgogne.fr
}

rappelle le rôle du lecteur qui assimile, s'approprie et travaille le texte poétique : « « Mi agradecimiento es enorme para cada una de las lectoras y lectores que hizo Miedo de ser escarcha suyo, para cada artista que decidió dialogar con él o darle nuevas formas ", Rodríguez David Eloy, Miedo de ser escarcha (édition actualisée), op. cit., p. 9.

76 «Cela étant, faire acte de poésie, c'est aujourd'hui faire un acte de résistance à l'avilissement de l'intériorité par des stéréotypes qui, sous prétexte de démocratie, stérilisent l'émotion et l'imagination tout en privant de sens la pensée. Un produit conçu pour tous est forcément un produit insignifiant selon les critères de la consommation. La poésie, donc, est d'emblée inconsommable parce qu'elle a besoin, comme l'amour, d'un effort d'attention pour qu'advienne le partage. Sa signification est dans la qualité qu'elle rend alors au langage et, par lui, à la relation humaine... ", Noël Bernard, La Place de l'autre, Euvres III, Paris, POL, 2013, p. 760.

77 "C’est pourquoi la poésie est un poste d'observation privilégié sur le langage en général, sur la pensée et sur la société [...] ", Meschonnic Henri, Célébration de la poésie, Paris, Verdier, 2001, quatrième de couverture.

78 Bernard Noël définit ainsi la poésie comme le « foyer de résistance de la langue vivante contre la langue consommée ", cité dans Meschonnic Henri, Célébration de la poésie, Paris, Verdier, 2001, p. 118.

79 Durant le premier semestre de l'année 2014, un atelier de traduction a été animé par un groupe d'étudiants de l'Université de Limoges autour des textes de José María Gómez Valero et David Eloy Rodríguez. L'anthologie ici présentée est ainsi redevable aux travaux et réflexions menés par R. Cayla, Z. Durand, C. Fourcade, M. Lacassaigne, C. Langlois, S. Llido, M. Mahadali, A. Parnoix, A. Strugeon, J. Stroubante et M. Veysset. Qu'ils soient ici vivement remerciés pour leur travail et enthousiasme. 


\section{Résumé}

Dans le panorama de la poésie espagnole contemporaine, il est des voix réfractaires organisées en " récommunes " qui, articulant le poétique et le politique, désertent les perspectives balisées d'une écriture aseptique, amnésique et auto-satisfaite. Le collectif La Palabra Itinerante en est. Créé en Andalousie en 1992, il travaille à différentes hybridations des formes d'art et devient le lieu où l'écriture se donne pour objectif l'agitation des consciences (" colectivo de agitación y expresión ») et la déconstruction des machines à discipliner. Cet article présente brièvement l'œuvre de trois poètes, José María Gómez Valero (Séville, 1976), David Eloy Rodríguez (Cáceres, 1976) et David Franco Monthiel (Cádiz, 1976), en mettant au jour quelques pratiques poétiques dominantes : le primat du commun, le détournement, l'écriture du slogan, les formes cursives et denses. Une anthologie de textes traduits complète cette présentation.

\section{Mots-clés}

Poésie espagnole contemporaine, poètes " de la conscience critique », écritures de la résistance.

\section{Resumen}

En el panorama actual de la poesía española, destacan unas nuevas voces refractarias organizadas en « recomunas " que, articulando lo poético y lo político, desertan las perspectivas balizadas de una escritura aséptica, amnésica y autosatisfecha. Entre estos grupos, encontramos el colectivo andaluz La Palabra Itinerante, formado a principios de los noventa, que trabaja las hibridaciones de diferentes artes y tiene como objetivo la agitación de las conciencias (" colectivo de agitación y expresión ") y la demolición de las máquinas de disciplinamiento. Este artículo presenta brevemente la obra de tres integrantes de esta plataforma: José María Gómez Valero (Sevilla, 1976), David Eloy Rodríguez (Cáceres, 1976) y David Franco Monthiel (Cádiz, 1976), poniendo de realce algunas de las prácticas y modalidades de escritura más sobresalientes: el alcance de lo común en una primera persona del plural, la tergiversación, la escritura del eslogan y las formas aforísticas. Se presenta a continuación una selección de textos traducidos al francés.

\section{Palabras claves}

Poesía española contemporánea, poetas " de la conciencia crítica ", La Palabra Itinerante, escrituras de la resistencia. 


\section{Anthologie de textes traduits}

\section{Textes de José María Gómez Valero}

QUÉ TRISTE LA VIDA

de una línea recta:

tan sola

$\tan$ recta

tan uniforme.

EL IDIOMA DEL LABERINTO

I

Nada se entiende en estos días:

¿No somos todos

extranjeros en la ciudad

del ruido y del dinero?

II

Frente a los signos vencidos,

el hambre de un lenguaje

diferente y crucial

que pudiera aprenderse

sin dificultad ni daño.

¡AGUa!, rogó el sediento.

$\mathrm{Y}$ al instante

lo colmaron de agasajos,

de medallas,

de aplausos,

de vítores.

¡Agua! ¡Agua!,

fue lo último que logró decir

el agonizante.

\section{PAtria}

Cuando le preguntaron al extranjero por su procedencia, éste señaló, uno a uno,

a todos los habitantes de la ciudad.

\section{CíRCULOS CONCÉNTRICOS}

El profesor dibujó

dos círculos perfectos

y seguidamente dijo:

¿veis?, como una rueda.

El niño inquirió:

sí, como unos ojos.

El profesor respondió:

no, como una rueda.
Quelle tristesse LA VIE

d'une ligne droite :

si seule

si droite

si uniforme.

LE LANGAGE DU LABYRINTHE

\section{I}

Plus rien n'est aujourd'hui compréhensible:

Ne sommes-nous pas tous

étrangers dans cette ville

du bruit et de l'argent?

II

Face aux signes vaincus,

la soif d'un langage

différent et crucial

qui pourrait s'apprendre

sans difficulté et sans peine.

À Borre !, supplia l'homme assoiffé.

Et sur-le-champ

on le couvrit de présents

de médailles,

d'applaudissements,

de vivats.

À boire! À boire!,

Ce furent les dernières paroles

de l'homme agonisant.

Patrie

Quand on demanda à l'étranger

son origine,

il montra du doigt, un à un,

tous les habitants de la ville.

\section{Cercles concentriques}

Le professeur dessina

deux cercles parfaits

puis ensuite il dit :

Vous voyez?, c'est comme une roue.

L'enfant demanda :

oui, comme des yeux, n'est-ce pas?

Le professeur répondit :

non, c'est comme une roue. 
Equipajes

Amar sin daño, amar sin dueño.

Saber reír, saber curar.

Sentir en cada paso

el frescor de la tierra

bajo la hojarasca.

Juegos REUNIDOS

Dados en blanco

para perder

cada partida

contigo.

Dados en blanco

y en el aire.

Apuntes para una biografía CUALQUiera

Nacer,

memorizar los signos,

ocupar una celda

en la intemperie.

Reconocer a tientas

el rigor de los límites,

los contornos del orden.

Asistir cada día

a lo pactado.

Mirar el agua,

saciarse en su sabor,

convivir con la sed.

Acatar los dictados de la norma,

eludir los dictados de la norma.

Jugar a cosas serias.

Mentir de corazón.

Arroparse sin sueño.

La noche,

los velos, los desvelos,

la voz

de la sólida sombra.

Despertar,

abrir los ojos,

ansiar el tiempo

en el que nada se derrumba.

\section{BAgAgES}

Aimer sans dommage

Aimer sans maître.

Savoir rire,

savoir guérir.

Sentir à chaque pas

la fraîcheur de la terre sous le feuillage.

JEUX RÉUNIS

Un dé aux faces blanches

pour perdre

chaque partie

avec toi.

Un dé aux faces blanches

et jeté dans les airs.

APOSTILLES POUR UNE BIOGRAPHIE ORDINAIRE

Naître,

mémoriser les signes,

occuper une cellule

exposée aux intempéries.

Reconnaître à tâtons,

l'âpreté des limites,

les contours de l'ordre.

Être témoin chaque jour

du pacte.

Observer l'eau,

se rassasier de sa saveur

cohabiter avec la soif.

Obéir aux lois de la norme,

échapper aux lois de la norme.

S'amuser de jeux sérieux.

Mentir de bon cœur.

S'endormir sans sommeil

La nuit,

somnolente, éveillée

la voix

d'une ombre solide.

Se réveiller,

ouvrir les yeux,

être avide d'un temps

où rien ne s'effondre. 


\section{Textes de David Eloy Rodríguez}

Marat-Sade, 1998

El problema ahora

es que hay muchos vigilantes

y pocos locos.

El problema ahora

es que la jaula está

en el interior del pájaro.

\section{Despacito y mala Letra}

Confiamos en la hierba

frente al empedrado:

no siempre ganará el más fuerte.

\section{Aquí todavía}

Nací en las ciudades.

Huí de la ciudad de mis padres.

Busqué personas con piel de arcilla, tuve un sombrero,

ascendí por mujeres de yedra, supe del milagro y la desolación.

La muerte que he visto

no ha ganado aún mis ojos.

Descubrí que la mejor forma de vivir era no trabajar para el enemigo.

\section{EL DESEO ES UN HUÉSPED}

Se entra en el amor con el bullicio de chiquillos que juegan en una piscina.

Se sale del amor con el silencio de dos viejos que miran en la playa la lenta asfixia de un ahogado.
Marat-Sade, 1998

Le problème à présent c'est qu'il y a trop de geôliers et peu de fous.

Le problème à présent c'est que la cage se trouve à l'intérieur de l'oiseau.

\section{Petit À Petit et en GRiffonnant}

Nous avons confiance en cette herbe face aux pavés :

parfois la victoire ne sera pas celle du plus fort.

\section{ICI ENCORE}

Je suis né dans les villes.

J'ai fui la ville de mes pères.

J'ai recherché des hommes à la peau d'argile, j'ai porté un chapeau,

j’ai grimpé sur des femmes de lierre,

j'ai connu le miracle et la désolation.

La mort que j'ai parfois croisée

n'a pas encore gagné mes yeux.

J'ai découvert que la meilleure façon de vivre était de ne pas servir l'ennemi.

\section{LE DÉSIR EST UN HÔTE}

On entre en amour

dans un brouhaha d'enfants

qui jouent dans une piscine.

On quitte l'amour

dans le silence de deux vieillards

qui regardent sur la plage

la lente asphyxie d'un noyé. 
LABOR

Yo traje a este sitio mi cuerpo y aquí lo desgasto en jornadas, aquí me esfuerzo de luna a luna hasta que la palabra descanso florece hermosísima en la boca.

El techo bajo el que nos guarecemos es provisional e inestable; en ocasiones confundimos todo esto con un hogar.

Conformamos una familia extrańa: hermanos bajo las luces permanentemente encendidas

de la videovigilancia, sacándole punta al tiempo

en una labor enhebrada por obediencias y desobediencias, sutiles percepciones, soledades y compañías, diálogos callados.

Vistos desde lejos parecemos granos de arena arrastrados por un viento inútil. "¿Y qué importa?",

nos decimos los unos a los otros.

Pero en los sueños murmuran sombras que nos interrogan y nos turban, que musitan: “¿Cómo se puede ser arena sin ser desierto, sin sufrir la sed?"

El jornal no paga la sangre de mis horas, su alto sacrificio.

En el trabajo está prohibido hablar. Pero yo hablo. Todos hablan.

\section{LÍNEAS DE FUGA}

Huir lejos del odio y sus madrigueras encendidos de pasión y búsquedas. Huir por desesperaciones y refugios con un equipaje de amor y desasosiego. Huir hacia una hora sin puntos cardinales, como equilibristas por el fino cordel de la cordura o como mendigos que persiguen un merecido corazón sobre la tierra. Huir guiados por brújulas rotas. Huir confiando en la fuga. Huir para encontrarnos.
TravaIL

J'ai porté ici mon corps

et c'est ici que je l'use du matin au soir, c'est ici que je me fatigue du soir au mouroir jusqu'à ce que le mot repos fleurisse, étincelant dans ma bouche.

Le toit sous lequel nous nous abritons est provisoire et instable ; parfois nous pensons à tort qu'il s'agit d'un chez-soi Nous formons une étrange famille : des compagnons sous les lumières sans cesse allumées

des caméras de surveillance, qui taillent le temps

dans un travail oscillant entre obéissance et désobéissance, des perceptions subtiles, entre solitude et compagnie, des dialogues en sourdine.

De loin nous ressemblons à des grains de sable, emportés par un vent inutile. Mais qu'importe?, nous disons-nous.

Cependant dans nos rêves des ombres murmurent, nous interrogent et nous troublent, elles chuchotent : Comment peut-on être un grain de sable, si l'on n'est pas un désert, si l'on n'éprouve pas la soif? Le salaire journalier ne paie pas le sang de mes heures, son très haut sacrifice.

Au travail il est interdit de parler.

Mais moi je parle. Nous parlons tous.

\section{LigneS DE FUITE}

Fuir loin de la haine et de ses tanières portés par la passion et la quête.

Fuir et aller de désespoirs en refuges avec pour seul viatique l'amour et le trouble. Fuir vers un temps sans points cardinaux comme un équilibriste sur la corde frêle de la sagesse ou comme des mendiants qui poursuivent un cœur bien mérité sur cette terre.

Fuir guidés par des boussoles brisées. Fuir et croire en la fuite. Fuir pour se retrouver. 
SOMOS ETERNOS EN CADA DECISIÓN

I

Es la tierra, son los lobos, es la luna.

Tus pies en este barro.

Tus pies. Este barro.

II

Ya casi no pisamos tierra, pisamos nombres, cifras, y eso no es caminar.

¡Tan lejos de tanto que está tan cerca!

Cada día acontece

la expulsión del paraíso.

III
NOUS SOMMES ÉTERNELS EN CHAQUE DÉCISION I

C'est la terre, ce sont les loups, c'est la lune.

Tes pieds dans la boue.

Tes pieds. La boue.

II

Il ne reste presque plus de terre à fouler, nous foulons des nombres, des chiffres, et ce n'est pas marcher que cela.

Si loin de tout ce qui est si proche!

Chaque jour a lieu

l'expulsion du paradis.

III

Hay que confirmar el mundo en todos sus [extremos], Il faut confirmer le monde en tous sens acariciar cada cosa caresser toute chose para comprobar que está en su sitio. pour s'assurer que tout est à sa place.

Destituidos del verbo libertad, despojados de vivencia, somos seres sin hogar posible, Destitué du verbe de la liberté Dépouillé de nos expériences perros famélicos que escarban, desesperados, des chiens faméliques qui fouillent, désespérément, en una sepultura.

la terre d'une sépulture.

\section{Textes de David Franco Monthiel}

LA SAJADURA DEL AFILADOR

Un puñado

de finas hebras

nos sostienen.

Una

fibra,

un

cordel,

un hilito,

sos-

tie-

nen

el orden.

Y sus diez millones cuatrocientos

treinta y cuatro cuchillos

romos.

Y sus trescientas treinta y cinco mil tijeras sin afilar.

\section{BAjo LOS ADOQUines, LOS ADOQUINES}

Sé utópica.

Y pide lo posible.

Aunque parezca

que está permitido.
L'INCISION DU RÉMOULEUR

Une poignée

de minces filaments

nous tiennent.

Une

fibre,

une

ficelle,

un simple fil,

sous-

tien-

nent

l'ordre.

Et ses dix millions quatre cent trente quatre couteaux émoussés.

Et ses trois cent trente cinq mille ciseaux non aiguisés.

SOUS LES PAVÉS, LES PAVÉS

Sois utopique.

Et demande ce qui est possible.

Même si cela

semble permis. 
NUNCA DIGAS NUNCA

Nunca sacrifiques los odios en las elecciones del miedo.

Nunca desmorones las tardes ante el eterno vacío de la abundancia. Nunca olvides

la soberanía de echar a andar, de ser camino, dolor que se afila. Identifica los huecos, la grieta, los deshilvanes del sucio traje que dicen toga de las mejores sedas. Mira sus párpados húmedos y los pantanos secos.

Nunca olvides la música de las trincheras. Nunca entierres el verso vidente de aún no estar cegados, el rescoldo métrico de una hoguera apagada. Nunca dejes de oír la sinfonía insurrecta en la profundidad, el rugido de las calles y barriadas. Nunca dejes de acariciar la piel que rechina contra el mundo.
IL NE FAUT JAMAIS DIRE JAMAIS

Ne sacrifie jamais les haines sur l'autel de la peur.

N'abat jamais les soirs

face à l'éternel vide de l'abondance.

N'oublie jamais,

la souveraineté du départ,

d'être un chemin, douleur affûtée.

Repère les déchirures, les fissures, les fils débâtis de l'habit encrassé

exhibé comme une toge faite des plus belles soies. Regarde ses paupières humides et les marais asséchés.

N'oublie jamais la musique des tranchées. N'enterre jamais le vers divinatoire, nous en serions aveuglés,

les ultimes braises métriques d'un bûcher éteint. Ne suspend pas l'écoute

de la symphonie insurgée des profondeurs, le rugissement de la rue et des quartiers, Ne retiens pas la caresse de la peau qui grince contre le monde. 
MuRo CON INSCRIPCIONES DESVAÍDAS

I

Leí y luché.

II. Cuestión DE TIEMPO

Piensa.

Luego, insiste.

III. ESTE ABRAZO

Cualquier cosa que destruyas nosotras la crearemos de nuevo.

IV. LO REAL NO ES L'OrÉAL

Teje un sueño

y cose con él la realidad.

V. TeORÍA DE LA DESOBEDIENCIA EN CUATRO PALABRAS

No. No. Y no.

VI. Change Weschel Valuta

No cambies de jefes.

Cambia de manera de vivir.

VII. NACIÓN

Múltiple, pequeña y librérrima.

VIII. We SHALL OVERCOME

- Venceremos.
UN MUR AUX INSCRIPTIONS EFFACÉES

I

J'ai lu et j'ai lutté.

II. QUESTION DE TEMPS

Pense.

Donc, insiste.

III. Cette accolade

Toute chose par toi détruite

nous la recréerons nouvellement.

IV. Le RÉEL N’EST PAS L’OrÉAL

Tisse un rêve

et couds de ce fil la réalité.

V. THÉORIE DE LA DÉSObÉISSANCE EN QUATRE мотS

Non. Non. Et non.

VI. Change Weschel Valuta

Ne change pas de chef.

Change de façon de vivre.

VII. NATION

Multiple, petite et archilibre.

VIII. We SHALL OVERCOME

- Nous triompherons.
Ven. Seremos.

IX. Estrategias Microfísicas

Ama cualquier conquista

por pequeña que sea,

por leve, por ínfima,

por imperceptible,

por microscópica,

pour cuántica que sea.

Ama cualquier conquista.

Buenos díAs, PEREZA

Imaginar la vida fuera de la noria.

Evitar las bridas, fichar con la lengua.

Oxidarse en convenios para todos.

Olvidar luchar por cada miga

de la hora del bocadillo. Subir la guardia.

Bajarse al moro, morar en lo desnudo.

Desvanecer las dudas. Los bretes.

Desanudar las cabezas del estómago.

Dejar de ser un hombre sin rostro.

Y empezar a tener rastro.
Viens. Nous ferons le triomphe.

IX. Stratégies microphysiques

Aime toute conquête

aussi petite soit-elle,

aussi légère, aussi infime,

aussi imperceptible,

aussi microscopique,

aussi quantique soit-elle.

Aime toute conquête.

\section{BONJOUR, PARESSE}

Imaginer la vie hors de la noria.

Eviter les brides, pointer avec la langue.

Se laisser oxyder dans des accords pour tous.

Renoncer à la lutte pour la moindre miette

de l'heure de la pause. Monter la garde.

Traficoter aux côtés de la nudité.

Dissiper les doutes. Les embarras.

Démêler les mots à l'estomac.

Cesser d'être un homme sans visage.

Pour enfin laisser trace et balisage. 